\title{
Interface electronic properties of heterojunctions based on nanocrystalline silicon
}

\author{
E.B. Kaganovich, S.I. Kirillova, E.G. Manoilov, V.E. Primachenko, S.V. Svechnikov \\ Institute of Semiconductor Physics of NASU, 45, prospect Nauki, 252028 Kiev, Ukraine
}

\begin{abstract}
For investigations of electronic properties of heterojunctions nanocrystalline Si film (nc-Si)/ monocrystalline $\mathrm{Si}(\mathrm{c}-\mathrm{Si}$ ) the technique of temperature dependencies of surface photovoltage was used. Two types of samples fabricated by laser ablation of c-Si target with deposition of nc-Si films onto substrates situated at a distance from the target and onto the plane of target were studied. The temperature dependencies of concentration of charge carriers captured in the traps in the heterojunction interface, and of distribution of density of surface electron states on energy were calculated. The connections between conditions of heterojunction fabrication and their electronic properties are clarified.
\end{abstract}

Keywords: silicon, nanocomposite, boundary electronic states, surface photovoltage.

Paper received 01.06.99; revised manuscript received 05.07.99; accepted for publication 12.07.99.

\section{Introduction}

The interest to electronic properties of interfaces between nanocrystalline silicon (nc-Si) and monocrystalline silicon (c-Si) is explained by the possibility of creation of light sources in visible region of the spectrum on the basis of $\mathrm{nc}-\mathrm{Si} / \mathrm{c}-$ Si heterostructures. The design of such sources are in initial stage [1-5]. It is suggested that in the structures nc-Si/c-Si the heterojunction is formed between the wide-bandgap nc$\mathrm{Si}$ and narrow-bandgap c-Si, and the expanded bandgap of $\mathrm{nc}-\mathrm{Si}$ is related to the quantum limitation of the charge carriers in the Si nanocrystals. Nanocrystalline silicon has the nanocomposite structure, containing Si nanocrystals in the matrices of oxide, nitride, silicon carbide, amorphous silicon, etc. Their nearest relative is the porous silicon (por-Si) representing a three-phase nanocomposite structure containing low-dimensional Si nanocrystals in the matrix of the porous silicon oxide. Recently some information about electronic properties of por-Si/c-Si structures were obtained during photovoltage studies [6-8]. To the best of our knowledge, the data about the electronic properties of the nc-Si/cSi structures are absent in literature. But such information are necessary for understanding the properties of these het- erojunctions and the designing of optoelectronic devices on their basis.

The objective of this paper is to investigate, using the technique of temperature dependencies of pulse photovoltage, the nc-Si/c-Si structures fabricated by pulse laser ablation of nc-Si thin films onto c-Si substrates. Since the boundary potential of the c-Si surface, $\varphi_{s}$, plays an important role in operation of the $\mathrm{nc}-\mathrm{Si} / \mathrm{c}-\mathrm{Si}$ structures, in this work the features of the temperature dependencies of $\varphi_{\mathrm{s}}$ in the nc-Si/ $\mathrm{c}-\mathrm{Si}$ structures formed at different conditions of deposition of thin nc-Si film onto the c-Si substrate of $p$-type conductivity $(p-\mathrm{Si})$ are investigated.

\section{Samples and experimental technique}

The boundary potential of the c-Si surface $\varphi_{s}$ (the bending of energy bands at the surface) was determined using the technique of capacitance photovoltage at high levels of generation charge carriers flattening the band bending at the silicon surface. For the measurements the capacitor was constructed: investigated sample nc-Si/c-Si - mica plate with deposited conductive layer of $\mathrm{SnO}_{2}$. Thin aluminum films deposited on the edges of the $p$-Si surface provided Ohmic 


\section{E.B. Kaganovich et al.: Interface electronic properties of heterojunctions...}

low-noise contacts at low temperatures. The sample was formed by the deposition onto the c-Si substrate of nc-Si film by the laser ablation technique using the set-up constructed on the basis of the standard vacuum post with residual pressure $10^{-3} \mathrm{~Pa}$ and pulse solid-state laser YAG: $\mathrm{Nd}^{+3}$ operating in the regime of modulated quality with the irradiation wavelength of $1.06 \mathrm{~mm}$, energy in the pulse $0.2 \mathrm{~J}$, pulse duration $10 \mathrm{nsec}$ and repetition frequency $25 \mathrm{~Hz}$ [9]. As a target p-Si (KDB-10) and $n$-Si (KEF-4.5) and substrates of $p$-Si (100) were used. Deposition was carried out onto substrates separated from the target, from the direct flow of particles of erosion flame and to the substrate situated in the plane of the target, from the reverse flow of particles, being turned back as a result of collisions with gas molecules in the chamber [10]. Sputtering was performed in the atmosphere of inert (helium, argon) or reactive (oxygen) gas varying its pressure from $10^{-2} \mathrm{~Pa}$ to $5 \cdot 10^{2} \mathrm{~Pa}$. After chemical and mechanical polishing, the substrates were treated in $49 \%$ solution of HF with subsequent rinsing in distilled water. The thickness of nc-Si films were about $100 \mathrm{~nm}$.

When the capacitor was illuminated by light flashes of the ISSh-100 flash lamp with $10 \mathrm{msec}$ duration, repetition frequency $1 \mathrm{~Hz}$ and intensity $10^{21}$ quanta $\cdot \mathrm{cm}^{-2} \cdot \mathrm{sec}^{-1}$, the photovoltage signal $V_{p h}$ appeared, which was recorded using the memory oscilloscope. The measurements of $V_{p h}$ were carried out in the cryostat (pressure $10^{-4} \mathrm{~Pa}$ ) when reducing the temperature $T$ from 300 to $100 \mathrm{~K}$. In order to obtain the magnitude of $\varphi_{S}$ the circuit calibration was done using the testing electrical pulse. The high intensity of the used light allowed to rectify the energy bands of c-Si during irradiation [11]. In several cases the magnitude of the photovoltage measured during the action of the first pulse of light was different from that at the second or any subsequent pulse due to a capture of non-equilibrium charge carriers at the surface traps during the first pulse. Because of this the sample after each measurement was heated to the temperature at which the release of these traps took place and then again was cooled in the darkness to the temperature of new measurements of $V_{p h}$ first and second values [12].

\section{Results and Discussion}

Below, the results of measuring temperature dependencies of interface potential $\varphi_{S}(T)$ for two sample sets (Fig. a, c) and results of calculating their interface electron states and traps capturing the charge carriers concentration (Fig. b, d) are presented. The first set of samples was fabricated using the direct flow of particles, and the second set was formed using the reverse one.

In the Figure (a) the temperature dependencies of the interface potential $\varphi_{S}(T)$ are shown for three structures from the first set, obtained by the ablation of $p$-Si target with deposition onto $p$-Si substrates. The structures differ by conditions of fabrication. The first one was formed in the helium atmosphere at the pressure $\sim 10^{-1} \mathrm{~Pa}$, the second one was fabricated at the residual pressure of the air in the vacuum chamber, i.e. in the vacuum $\sim 10^{-3} \mathrm{~Pa}$, the third one was fabricated in the oxygen atmosphere at the pressure $6 \mathrm{~Pa}$. The first and second nc-Si films did not show the photoluminescense (PL) in the visible part of spectrum, but in the third one the PL with a broad maximum in the range 400-800 nm was observed. As our previous measurements of absorption spectra have shown [9], the size of the nanocrystals only in the third film were less than $3 \mathrm{~nm}$. The numbers of the curves in the Figure (a) correspond to the types of structures. In the same Figure (a) the dependence of $\varphi_{S}(T)$ for $p$-Si substrate before the deposition of nc-Si film was shown (curve 4).

It is seen from the Figure (a) that, as it is usually observed in the $p$-type samples, the sign of photovoltage is positive, the depletion of the $p$-Si surface takes place for the majority carriers, holes, i.e. the energy bands are bent down. With decreasing temperature the value of $\varphi_{s}$ increases for all curves 1 to 4 , which is related to filling interface electron states (IES) of the substrate by holes when the Fermi level in $p$-Si is displaced to the valence band with temperature variation. From plots $\varphi_{S}(T)$ it is possible to calculate the density of IES in the probed by the Fermi level section of the bandgap in the surface of $p$-Si [13]. It was found that for free $p$-Si substrate the maximum concentration of IES, equal to $N_{s \text { max }}=8 \cdot 10^{11} \mathrm{~cm}^{-2} \mathrm{eV}^{-1}$ is situated by $0.18 \mathrm{eV}$ below the middle of the bandgap $E_{i}\left(E_{i}-0.18 \mathrm{eV}\right)$. For the structure of the third type $N_{s \text { max }}=4 \cdot 10^{11} \mathrm{~cm}^{-2} \mathrm{eV}^{-1}$ at $E_{i}-0.20 \mathrm{eV}$. For the first and second structures, it is characteristic that in these structures the position of the Fermi level in the interface is practically unchanged with changing temperature. It is fixed near $E_{i}$ by high concentration of IES $\left(N_{s}>10^{12} \mathrm{~cm}^{-2} \mathrm{eV}^{-1}\right)$.

The dependencies $\varphi_{s}(T)$ measured during the second pulse of light (curves $1^{\mathrm{I}}$ to $4^{\mathrm{I}}$ ) at $T<180-200 \mathrm{~K}$ are below the curves measured during the first pulse. Such a difference is due to the capture of non-equilibrium electrons in the interface traps during the illumination by the first pulse, which forms the photomemory of potential $\varphi_{S}$ [12]. Note that the most prominent effect is observed for the structures of the first and second types, which are characterized also by the greatest magnitudes of $\varphi_{s}$. From the data of the observed photomemory effect the temperature dependencies of the number of trapped electrons $N(T)$ (Figure (b)) are calculated [14]. Since the traps are completely filled by electrons just during the first pulse of light, the dependencies $N(T)$ represent also the temperature dependencies of trap concentration. The increase of $N$ with decreasing temperature is related to involvement of more shallow traps, located closer to the conduction band. It is seen from Figure (b) that at low temperatures the structures of the first and second types are characterized by the greatest capture of electrons $\left(\sim 4 \cdot 10^{10} \mathrm{~cm}^{-2}\right)$ at shallow traps.

In Figure (c) are presented the dependencies $\varphi_{S}(T)$ for five structures of the second set of samples, obtained by ablation of $p$-and $n$-type Si targets (curves 2, 3, 4 and curves 1,5 , respectively) with deposition onto $p$-Si substrates of nc-Si films in the atmosphere of He (curves 1,2) and $\mathrm{Ar}$ (curves $3-5$ ) at pressures $3 \cdot 10^{2} \mathrm{~Pa}$ and $3 \cdot 10^{1} \mathrm{~Pa}$, respectively. Thicknesses of all films, except 4 , are $100 \mathrm{~nm}$, and the thickness of the film 4 is $10 \mathrm{~nm}$. All nc-Si films had visible PL. As it is seen from the Figure (c), this set of samples is characterized by small values of $\varphi_{s}(-50 \div 150 \mathrm{mV})$, both positive, and negative, and by the absence of photomemory 


\section{E.B. Kaganovich et al.: Interface electronic properties of heterojunctions...}
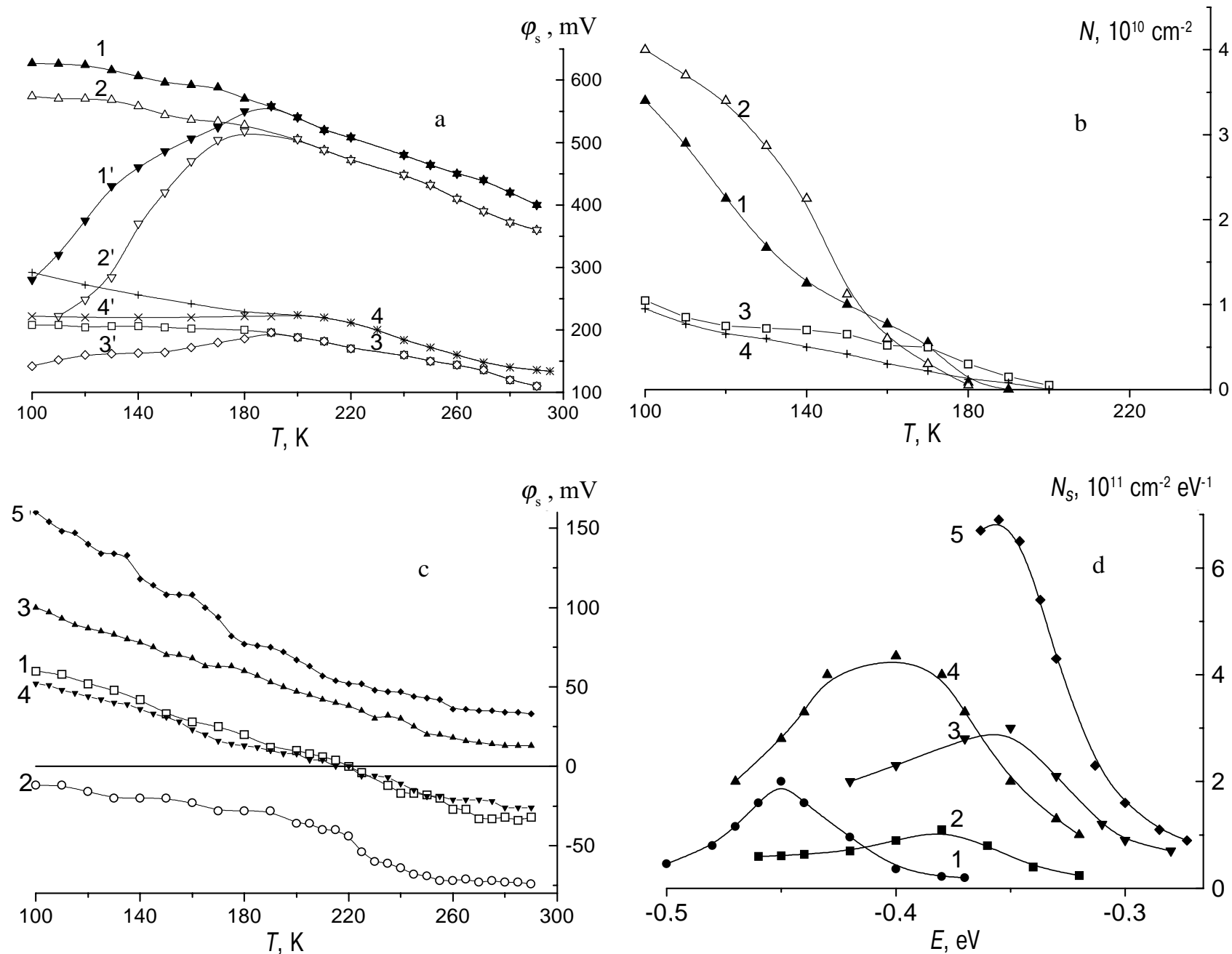

Figure. Temperature dependencies of interface potential $\varphi_{\mathrm{s}}$ of $p$-Si for the first (a) and second (c) set of samples nc-Si $/ p$-Si, and for the first set of samples the dependence of concentration $N$ of interface electron traps on temperature (b) and for the second set the dependence of IES density on energy $E$ below the middle of the bandgap (d). The first set of samples: 1 - He, $10^{-1} \mathrm{~Pa} ; 2$ - vacuum, $10^{-3} \mathrm{~Pa} ; 3$ - oxygen, $6 \mathrm{~Pa}$. The second set of samples for $(n, p)$ nc-Si films on $p$-Si: 1 - $n$, He; 2 - $p$, He; 3 - $p$, Ar; 4 - $p$, Ar, 10 nm; 5 - $n$, Ar.

Table. Interface electronic properties of heterojunctions nanocrystalline silicon/ monosilicon

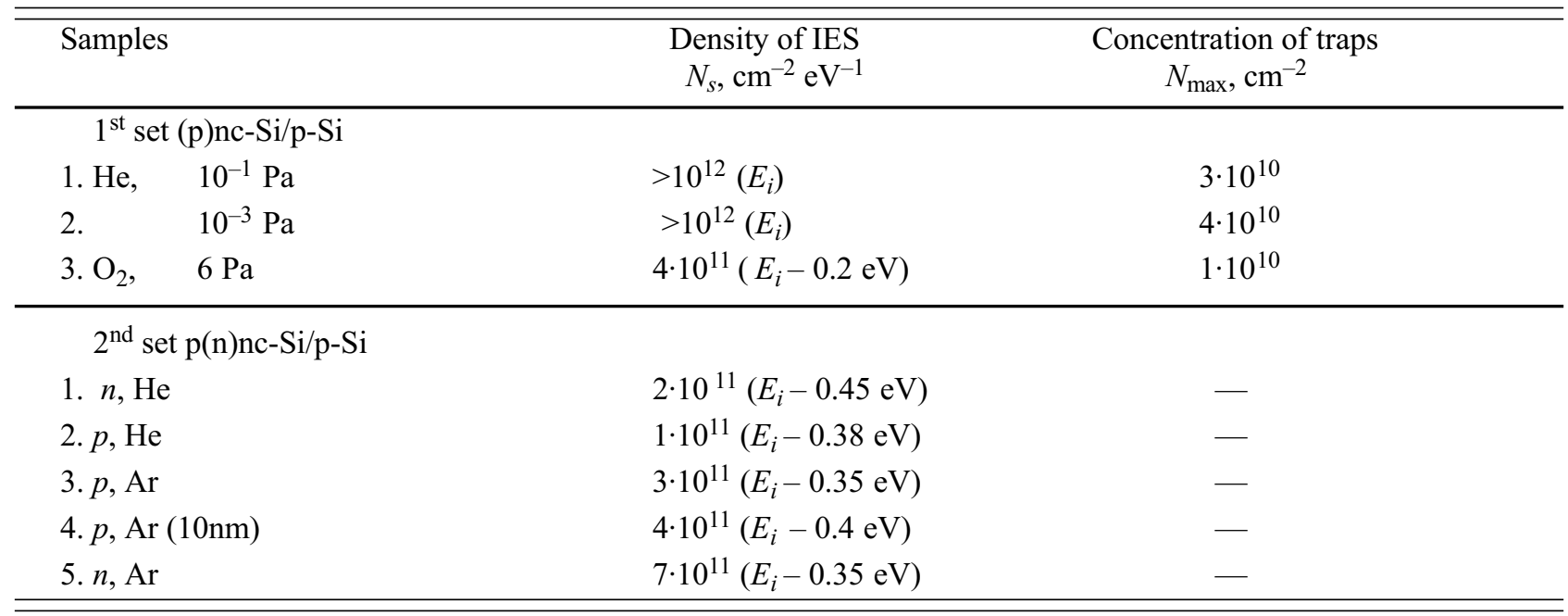

Note. Deposition for the first set of samples was performed from the direct particle flow in the flame, for the second set it was from the reverse flow. The number of the sample (number of the curve in the figure), type of conductivity of the target, gas in the laser ablation chamber are indicated. 


\section{E.B. Kaganovich et al.: Interface electronic properties of heterojunctions...}

effect. The calculation of energy distribution of the IES density below the midgap is presented in Figure (d). It showed at the level $E_{i}-(0.3-0.5) \mathrm{eV}$ the dependence of the IES density on the type of sputtered c-Si target (curves 1,2), on the type of inert gas Ar or He (curves 2, 3 and 1, 5), and on the thickness of deposited film (curves 3,4). The maximum densities of IES reach the values $N_{s \max }=(1-7) 10^{11} \mathrm{~cm}^{-}$ $2 \mathrm{eV}^{-1}$.

The obtained results are summarized in the Table. It can be seen from the Table that the interface electron properties of heterojunctions nanocrystalline silicon/monocryctalline silicon, formed by the laser ablation are sensitive to the conditions of fabrication. The measurement of temperature dependencies of the interface potential, calculation of densities of interface electron states and interface traps, capturing the nonequilibrium charge carriers make it possible to conclude the following. The highest $\left(>10^{12} \mathrm{~cm}^{-2} \mathrm{eV}^{-1}\right)$ density of IES is observed in the structures the films of which are fabricated during the deposition from the direct particle flow of the erosion flame in vacuum or in the inert gas at low pressures. Under the same conditions of film fabrication the highest $\left(\sim 4 \cdot 10^{10} \mathrm{~cm}^{-2}\right)$ density of traps capturing the nonequilibrium charge carriers is generated. It is seen from Figure (b) that these traps are mostly shallow, capturing the nonequilibrium carriers at lower temperatures. During the particle deposition from the direct flow at higher pressure in the oxygen atmosphere both IES density and concentration of shallow traps is reduced. This reduction is related to the interaction of particles sputtered by the laser beam with the oxygen molecules, as a result, their energy becomes lower and formation of the nc-Si film on the $p$-Si substrate takes place in conditions of creation of lesser number of defects in the substrate. Besides, oxidation of the substrate and of deposited particles may play a definite role in reduction of IES density.

The electron microscopy confirmed that nc-Si films fabricated in deposition from the reverse particle flow in the inert ambient are more uniform as compared to the films obtained in the direct particle flow. In the reverse flow their energy is greatly reduced due to interaction with the gas molecules. This results in the formation of nc-Si films with slightly poorer adhesion to the surface, but more uniform and with lower defect concentration in the film-substrate interface. This is confirmed by the data obtained in the second set of samples. It is seen from the Table, that the IES density for nc-Si film formation in the reverse particle flow is as a rule lower than that for the films from the direct flow at lower gas pressures. Besides, in formation of the nc-Si/p$\mathrm{Si}$ systems from the reverse particle flow the traps for nonequilibrium charge carriers are not created. It should be noted that in the second set of samples the IES density is lower for structure fabrication at higher pressure of inert gas (helium as compared to argon), and also is lower for the structures obtained at other equal conditions for the use of isotype targets and substrates.

These results are used for the control by the spectrum of local states of heterojunctions fabricated on the basis of nanocrystalline silicon and for revealing of their role in electron, photoelectron, electroluminescense properties of these heterojunctions.

\section{References}

1. Dimaria D.J., Kirtley J.R., Pakulis E.J. et al // J. Appl. Phys. 1984. v. 56. N2. P 401.

2. Qin G.G., Li A.P., Zhang B.R., Li B.C. // J. Appl. Phys. 1995. v. 78. N3. P 2006.

3. Yuan. J., Haneman D. // Appl. Phys. Lett. 1995. v. 67. N22. P 3328.

4. Song H.-Z., Bao X.-M., Li N.-S., Zhang J.-Y. // J.Appl. Phys. 1997. v. 82. N8. P 4028

5. Lalic N., Linnros J. // Proceedings E-MRS-98. Symposium B Manuscript:B-11 | P. | 3

6. Matveeva A.B., Konstantinova E.A., Timoshenko V.Yu., Kashkarov P.K. // FTP. 1995 v.29. ser.12 p.2180 (in Russian).

7. Timoshenko V.Yu., Konstantinova E.A., Ditrich T. // FTP 1998 v.32 No.5 p.613 (in Russian).

8. Bursstein L., Shapira Y., Partee J. // Phys. Rev. B. 1997. v. 55. N4. P. R 1930.

9. Indutnyi I.Z., Kaganovich E.B., Manoilov et al.// Ukr. Fiz. Zhurn 1997 v.42, No.1, p.85.

10. Movtchan I.A., Dreyfus R.W., Marine W. et al. // Thin Sol. Films. 1995. v. 255. P 286.

11. Sachenko A.V., Snitko O.V.// Fotoeffecty v pripoverkhnostnykh sloyakh poluprovodnikov (Photoeffects in the surface layers of semiconductors). Kiev: Nauk. Dumka, 1984. P.232 (in Russian).

12. Primachenko V.E., Snitko O.V. // Fizika legirovannoi metallami poverkhnosti poluprovodnikov (Physics of semiconductor surface doped by metals) Kiev: Nauk. Dumka, 1988. P.232 (in Russian).

13. Venger E.F., Kirilova S.I., Primachenko V.E., Chernobai V.A. // Ukr. Fiz. Zhurn. 1997, v.42, No.11/12 p.1333 (in Russian).

14. Kirilova S.I., Primachenko V.E., Chernobai V.A. // Optoelectronika $i$ poluprovodnikovaya tekhnika. 1991. No.21. p.60. 